\title{
Linear Quadratic Mean Field Game with Control Input Constraint
}

\author{
Ying $\mathrm{Hu}^{a}$, Jianhui Huang ${ }^{b}$, Xun $\mathrm{Li}^{c, *}$ \\ ${ }^{a}$ IRMAR, Université Rennes 1, Campus de Beaulieu, 35042 Rennes Cedex, France \\ ${ }^{b}$ Department of Applied Mathematics, The Hong Kong Polytechnic University, Hong Kong \\ ${ }^{c}$ Department of Applied Mathematics, The Hong Kong Polytechnic University, Hong Kong
}

September 17, 2018

\begin{abstract}
In this paper, we study a class of linear-quadratic (LQ) mean-field games in which the individual control process is constrained in a closed convex subset $\Gamma$ of full space $\mathbb{R}^{m}$. The decentralized strategies and consistency condition are represented by a class of mean-field forward-backward stochastic differential equation (MF-FBSDE) with projection operators on $\Gamma$. The wellposedness of consistency condition system is obtained using the monotonicity condition method. The related $\epsilon$-Nash equilibrium property is also verified.
\end{abstract}

Key words: $\epsilon$-Nash equilibrium, Mean-field forward-backward stochastic differential equation (MF-FBSDE), Linear-quadratic constrained control, Projection, Monotonic condition.

AMS Subject Classification: 60H10, 60H30, 91A10, 91A25, 93E20

\section{Introduction}

Our starting point comes from the recently well-studied mean-field games (MFGs) for largepopulation system. The large-population system arises naturally in various fields such as economics, engineering, social science and operational research, etc. The most salient feature of large-population system is the existence of a large number of individually negligible agents (or players) which are interrelated in their dynamics and (or) cost functionals via the state-average (in linear case) or more generally, the generated empirical measure over the whole population (in nonlinear case). Because of this highly complicated coupling feature, it is intractable for a given agent to employ the centralized optimization strategies based on the information of all its peers in large-population system. Actually, this will bring considerably high computational complexity in a large-scale manner. Alternatively, one reasonable and practical direction is to investigate the related decentralized strategies based on local information only. By local information, we mean that the related strategies should be designed upon the individual state (or, random noise) of given agent together with some mass-effect quantities which can be computed in off-line manner.

\footnotetext{
${ }^{1}$ Corresponding author: Ying Hu (Ying.Hu@univ-rennes1.fr)

${ }^{2}$ The work of Ying Hu is supported by Lebesgue center of mathematics "Investissements d'avenir" program - ANR-11LABX-0020-01, by ANR-15-CE05-0024-02 and by ANR-MFG; The work of James Jianhui Huang is supported by PolyU G-YL04, RGC Grant 502412, 15300514; The work of Xun Li is supported by PolyU G-UA4N, Hong Kong RGC under grants 15224215 and 15255416.

E-mail addresses: Ying.Hu@univ-rennes1.fr (Ying Hu); majhuang@polyu.edu.hk (Jianhui Huang); malixun@polyu.edu.hk (Xun Li).
} 
Along this research direction, one efficient and tractable methodology leading to decentralized strategies is the MFGs which generally lead to a coupled system of HJB equation and FokkerPlanck (FP) equation in nonlinear case. In principle, the procedure of MFGs consists of the following four main steps (see [13], 11, [10], [11, [4], [17, etc): in Step 1, it is necessary to analyze the asymptotic behavior of state-average when the agent number $N$ tends to infinity and introduce the related state-average limiting term. Of course, this limiting term is undetermined at this moment, thus it should be treated as some exogenous "frozen" term; Step 2 turns to study the related limiting optimization problem (which is also called auxiliary or tracking problem) by adopting the state-average instead of its frozen limit term. The initial high-coupled optimization problems of all agents are thus decoupled and only parameterized by this generic frozen limit. The related decentralized optimal strategy can be analyzed using standard control techniques such as dynamic programming principle (DPP) or stochastic maximum principle (SMP) (see e.g., [19]). As a result, some HJB equation (due to DPP) or Hamiltonian system (due to SMP) will be obtained to characterize this decentralized optimality; Step 3 aims to determine the frozen state-average limit by some consistency condition: when applying the optimal decentralized strategies derived in Step 2, the state-average limit should be reproduced as the agents number tends to infinity. Accordingly, some fixed-point analysis should be applied here and some FP equation will be introduced and coupled with the HJB equation in Step 2. As the necessary verification, Step 4 will show that the derived decentralized strategies should possess the $\epsilon$-Nash equilibrium properties. A comprehensive survey of MFG can be found in [3].

For further analysis of MFGs, the interested readers may refer to 6 for a survey of mean-field games focusing on the partial differential equation aspect and related real applications; [1] for more recent MFG studies and the related mean-field type control; [4 for the probabilistic analysis of a large class of stochastic differential games for which the interaction between the players is of mean-field type; [5] for the mean-field game where considerable interrelated banks share the system risk and common noise; [16] for a class of risk-sensitive mean-field stochastic differential games; [12] for MFGs with nonlinear diffusion dynamics and their relations to McKean-Vlasov particle system. It is remarkable that there exists a substantial literature body to the study of MFGs in linear-quadratic (LQ) framework. Here, we mention a few of them which are more relevant to our current work: 9] the mean-field LQ games with a major player and a large number of minor players, [1] the mean-field LQ games with non-uniform agents through the state-aggregation by empirical distribution, [14 the mean-field LQ mixed games with continuumparameterized minor players.

In this paper, we discuss the linear-quadratic (LQ) mean-field game where the individual control is constrained in a closed convex set $\Gamma$ of full space: $\Gamma \subset \mathbb{R}^{m}$. The LQ problems with control constraint arise naturally from various practical applications. For instance, the no-shorting constraint in portfolio selection leads to the LQ control with positive control $\left(\Gamma=\mathbb{R}_{+}^{m}\right.$, the positive orthant). Moreover, due to general market accessibility constraint, it is also interesting to study the LQ control with more general closed convex cone constraint (see [7]). As a response, this paper investigates the LQ dynamic game of large-population system with general closed convex control constraint. Our investigation is mainly sketched as follows. First, applying the maximum principle, the optimal decentralized response is characterized through some Hamiltonian system with projection operator upon the constrained set $\Gamma$. Second, the consistency condition system is connected to the well-posedness of some mean-field forward-backward stochastic differential equation (MF-FBSDE). Next, we present some monotonicity condition of this MF-FBSDE to obtain its uniqueness and existence. Last, the related approximate Nash equilibrium property is also verified. We derive the MFG strategy in its open-loop manner. Consequently, the approximate Nash equilibrium property is verified under the open-loop strategies perturbation and some estimates of forward-backward SDE are involved. In addition, all agents are set to be statistically identical thus the limiting control problem and fixed-point arguments are given 
for a representative agent. In case the agents are heterogeneous with different parameters, the similar procedure to MFG strategies can be proceeded via the introduction of index indicator and empirical state-average statistics.

The reminder of this paper is structured as follows: Section 2 formulates the LQ MFGs with control constraint. The decentralized strategies are derived with the help of a forward-backward SDE with projection operators. The consistency condition is also established. Section 3 verifies the $\epsilon$-Nash equilibrium of the decentralized strategies. Section 4 is appendix.

\section{Mean-Field LQG Games with Control Constraint}

Throughout this paper, we denote the $k$-dimensional Euclidean space by $\mathbb{R}^{k}$ with standard Euclidean norm $|\cdot|$ and standard Euclidean inner product $\langle\cdot, \cdot\rangle$. The transpose of a vector (or matrix) $x$ is denoted by $x^{T}$. $\operatorname{Tr}(A)$ denotes the trace of a square matrix $A$. Let $\mathbb{R}^{m \times n}$ be the Hilbert space consisting of all $(m \times n)$-matrices with the inner product $\langle A, B\rangle:=\operatorname{Tr}\left(A B^{T}\right)$ and the norm $|A|:=\langle A, A\rangle^{\frac{1}{2}}$. Denote the set of symmetric $k \times k$ matrices with real elements by $S^{k}$. If $M \in S^{k}$ is positive (semi)definite, we write $M>(\geq) 0 . L^{\infty}\left(0, T ; \mathbb{R}^{k}\right)$ is the space of uniformly bounded $\mathbb{R}^{k}$-valued functions. If $M(\cdot) \in L^{\infty}\left(0, T ; S^{k}\right)$ and $M(t)>(\geq) 0$ for all $t \in[0, T]$, we say that $M(\cdot)$ is positive (semi) definite, which is denoted by $M(\cdot)>(\geq) 0 . L^{2}\left(0, T ; \mathbb{R}^{k}\right)$ is the space of all $\mathbb{R}^{k}$-valued functions satisfying $\int_{0}^{T}|x(t)|^{2} d t<\infty$.

Consider a finite time horizon $[0, T]$ for fixed $T>0$. We assume $\left(\Omega, \mathcal{F},\left\{\mathcal{F}_{t}\right\}_{0 \leq t \leq T}, P\right)$ is a complete, filtered probability space on which a standard $N$-dimensional Brownian motion $\left\{W_{i}(t), 1 \leq i \leq N\right\}_{0 \leq t \leq T}$ is defined. For given filtration $\mathbb{F}=\left\{\mathcal{F}_{t}\right\}_{0 \leq t \leq T}$, let $L_{\mathbb{F}}^{2}\left(0, T ; \mathbb{R}^{k}\right)$ denote the space of all $\mathcal{F}_{t}$-progressively measurable $\mathbb{R}^{k}$-valued processes satisfying $\mathbb{E} \int_{0}^{T}|x(t)|^{2} d t<\infty$. Let $L_{\mathbb{F}}^{2, \mathcal{E}_{0}}\left(0, T ; \mathbb{R}^{k}\right) \subset L_{\mathbb{F}}^{2}\left(0, T ; \mathbb{R}^{k}\right)$ the subspace satisfying $\mathbb{E} x_{t} \equiv 0$ for $x . \in L_{\mathbb{F}}^{2, \mathcal{E}_{0}}\left(0, T ; \mathbb{R}^{k}\right)$.

Now let us consider a large-population system with $N$ weakly-coupled negligible agents $\left\{\mathcal{A}_{i}\right\}_{1 \leq i \leq N}$. The state $x^{i}$ for each $\mathcal{A}_{i}$ satisfies the following controlled linear stochastic system:

$$
\left\{\begin{aligned}
d x^{i}(t)= & {\left[A(t) x^{i}(t)+B(t) u_{i}(t)+F(t) x^{(N)}(t)+b(t)\right] d t } \\
& +\left[D(t) u_{i}(t)+\sigma(t)\right] d W_{i}(t), \\
x^{i}(0)= & x \in \mathbb{R}^{n}
\end{aligned}\right.
$$

where $x^{(N)}(\cdot)=\frac{1}{N} \sum_{i=1}^{N} x^{i}(\cdot)$ is the state-average, $(A(\cdot), B(\cdot), F(\cdot), b(\cdot) ; D(\cdot), \sigma(\cdot))$ are matrixvalued functions with appropriate dimensions to be identify soon. For sake of presentation, we set all agents are homogeneous or statistically symmetric with same coefficients $(A, B, F, b ; D, \sigma)$ and deterministic initial states $x$.

Now we identify the information structure of large population system: $\mathbb{F}^{i}=\left\{\mathcal{F}_{t}^{i}\right\}_{0 \leq t \leq T}$ is the natural filtration generated by $\left\{W_{i}(t), 0 \leq t \leq T\right\}$ and augmented by all $P$-null sets in $\mathcal{F} . \mathbb{F}=\left\{\mathcal{F}_{t}\right\}_{0 \leq t \leq T}$ is the natural filtration generated by $\left\{W_{i}(t), 1 \leq i \leq N, 0 \leq t \leq T\right\}$ and augmented by all $P$-null sets in $\mathcal{F}$. Thus, $\mathbb{F}^{i}$ is the individual decentralized information of $i^{t h}$ Brownian motion while $\mathbb{F}$ is the centralized information driven by all Brownian motion components. Note that the heterogeneous noise $W_{i}$ is specific for individual agent $\mathcal{A}_{i}$ but $x^{i}(t)$ is adapted to $\mathcal{F}_{t}$ instead of $\mathcal{F}_{t}^{i}$ due to the coupling state-average $x^{(N)}$.

The admissible control $u_{i} \in \mathcal{U}_{a d}^{c}$ where the admissible control set $\mathcal{U}_{a d}^{c}$ is defined as

$$
\mathcal{U}_{a d}^{c}:=\left\{u_{i}(\cdot) \mid u_{i}(\cdot) \in L_{\mathbb{F}}^{2}(0, T ; \Gamma)\right\}, 1 \leq i \leq N,
$$

where $\Gamma \subset \mathbb{R}^{m}$ is a closed convex set. Typical examples of such set is $\Gamma=\mathbb{R}_{+}^{m}$ which represents the positive control. Moreover, we can also define decentralized control as $u_{i} \in \mathcal{U}_{a d}^{d, i}$, where the 
admissible control set $\mathcal{U}_{a d}^{d, i}$ is defined as

$$
\mathcal{U}_{a d}^{d, i}:=\left\{u_{i}(\cdot) \mid u_{i}(\cdot) \in L_{\mathbb{F}^{i}}^{2}(0, T ; \Gamma)\right\}, 1 \leq i \leq N .
$$

Note that both $\mathcal{U}_{a d}^{d, i}$ and $\mathcal{U}_{a d}^{c}$ are defined in open-loop sense. Let $u=\left(u_{1}, \cdots, u_{i}, \cdots, u_{N}\right)$ denote the set of control strategies of all $N$ agents and $u_{-i}=\left(u_{1}, \cdots, u_{i-1}, u_{i+1}, \cdots, u_{N}\right)$ denote the control strategies set except the $i^{t h}$ agent $\mathcal{A}_{i}$. Introduce the cost functional of $\mathcal{A}_{i}$ as

$$
\begin{aligned}
\mathcal{J}_{i}\left(u_{i}, u_{-i}\right)= & \frac{1}{2} \mathbb{E}\left[\int_{0}^{T}\left\langle Q(t)\left(x^{i}(t)-x^{(N)}(t)\right), x^{i}(t)-x^{(N)}(t)\right\rangle\right. \\
& \left.+\left\langle R(t) u_{i}(t), u_{i}(t)\right\rangle d t+\left\langle G\left(x^{i}(T)-x^{(N)}(T)\right), x^{i}(T)-x^{(N)}(T)\right\rangle\right] .
\end{aligned}
$$

original cost

We impose the following assumptions:

(H1) $A(\cdot), F(\cdot) \in L^{\infty}\left(0, T ; S^{n}\right), B(\cdot), D(\cdot) \in L^{\infty}\left(0, T ; \mathbb{R}^{n \times m}\right), b(\cdot), \sigma(\cdot) \in L^{\infty}\left(0, T ; \mathbb{R}^{n}\right)$;

(H2) $Q(\cdot) \in L^{\infty}\left(0, T ; S^{n}\right), Q(\cdot) \geq 0, R(\cdot) \in L^{\infty}\left(0, T ; S^{m}\right), R(\cdot)>0$ and $R^{-1}(\cdot) \in L^{\infty}\left(0, T ; S^{m}\right)$, $G \in S^{n}, G \geq 0$.

It follows that (11) admits a unique solution $x^{i}(\cdot) \in L_{\mathbb{F}}^{2}\left(0, T ; \mathbb{R}^{n}\right)$ under admissible control $u_{i}$ with (H1), (H2). Now, we formulate the large population LQG games with control constraint (CC).

Problem (CC). Find an open-loop Nash equilibrium strategies set $\bar{u}=\left(\bar{u}_{1}, \bar{u}_{2}, \cdots, \bar{u}_{N}\right)$ satisfying

$$
\mathcal{J}_{i}\left(\bar{u}_{i}(\cdot), \bar{u}_{-i}(\cdot)\right)=\inf _{u_{i}(\cdot) \in \mathcal{U}_{a d}^{c}} \mathcal{J}_{i}\left(u_{i}(\cdot), \bar{u}_{-i}(\cdot)\right)
$$

where $\bar{u}_{-i}$ represents $\left(\bar{u}_{1}, \cdots, \bar{u}_{i-1}, \bar{u}_{i+1}, \cdots, \bar{u}_{N}\right)$, the strategies of all agents except $\mathcal{A}_{i}$.

The study of $(\mathbf{C C})$ is of heavy computational burden due to the highly-complicated coupling structure among these agents. Alternatively, one efficient method to search the approximate Nash equilibrium is the mean-field game theory, which bridges the "centralized" LQG games to the limiting LQG control problems, as the number of agents tends to infinity. To this end, we need to construct some auxiliary control problem using the frozen state-average limit. Based on it, we can find the decentralized strategies by consistency condition. More details are given below. Introduce the following auxiliary problem for $\mathcal{A}_{i}$ :

$$
\left\{\begin{aligned}
d x^{i}(t)= & {\left[A(t) x^{i}(t)+B(t) u_{i}(t)+F(t) z(t)+b(t)\right] d t } \\
& +\left[D(t) u_{i}(t)+\sigma(t)\right] d W_{i}(t), \\
x^{i}(0)= & x \in \mathbb{R}^{n}
\end{aligned}\right.
$$

and limiting cost functional is given by

$$
\begin{aligned}
J_{i}\left(u_{i}\right)= & \frac{1}{2} \mathbb{E}\left[\int_{0}^{T}\left\langle Q(t)\left(x^{i}(t)-z(t)\right), x^{i}(t)-z(t)\right\rangle\right. \\
& \left.+\left\langle R(t) u_{i}(t), u_{i}(t)\right\rangle d t+\left\langle G\left(x^{i}(T)-z(T)\right), x^{i}(T)-z(T)\right\rangle\right] .
\end{aligned}
$$

Now we formulate the following limiting stochastic optimal control (SOC) problem with control constraint (LCC).

Problem (LCC). For the $i^{\text {th }}$ agent, $i=1,2, \cdots, N$, find $u_{i}^{*}(\cdot) \in \mathcal{U}_{\text {ad }}^{d, i}$ satisfying

$$
J_{i}\left(u_{i}^{*}(\cdot)\right)=\inf _{u_{i}(\cdot) \in \mathcal{U}_{a d}^{d, i}} J_{i}\left(u_{i}(\cdot)\right) .
$$


Then $u_{i}^{*}(\cdot)$ is called a decentralized optimal control for Problem (LCC). Note that the cost functional is strictly convex and coercive thus it admits a unique optimal control $u_{i}^{*}$. Now we apply the maximum principle method to characterize $u_{i}^{*}$ with the optimal state $x^{i, *}$. First, introduce the following adjoint process

$$
\left\{\begin{aligned}
d p^{i} & =-\left[A^{T} p^{i}-Q\left(x^{i, *}-z\right)\right] d t+q^{i} d W_{i}(t), \\
p^{i}(T) & =-G\left(x^{i, *}(T)-z(T)\right) .
\end{aligned}\right.
$$

Applying the maximum principle, the Hamiltonian function can be expressed by

$$
\begin{aligned}
H^{i} & =H^{i}\left(t, p^{i}, q^{i}, x^{i}, u_{i}\right)=\left\langle p^{i}, A x^{i}+B u_{i}+F z+b\right\rangle \\
& +\left\langle q^{i}, D u_{i}+\sigma\right\rangle-\frac{1}{2}\left\langle Q\left(x^{i}-z\right), x^{i}-z\right\rangle-\frac{1}{2}\left\langle R u_{i}, u_{i}\right\rangle .
\end{aligned}
$$

Since $\Gamma$ is a closed convex set, then maximum principle reads as the following local form

$$
\left\langle\frac{\partial H^{i}}{\partial u_{i}}\left(t, p^{i, *}, q^{i, *}, x^{i, *}, u^{i, *}\right), u-u^{i, *}\right\rangle \leq 0, \quad \text { for all } u \in \Gamma \text {, a.e. } t \in[0, T], \mathbb{P}-\text { a.s. }
$$

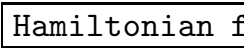

Noticing (44), then (5) yields that

$$
\left\langle B^{T} p^{i, *}+D^{T} q^{i, *}-R u^{i, *}, u-u^{i, *}\right\rangle \leq 0, \text { for all } u \in \Gamma \text {, a.e. } t \in[0, T], \mathbb{P}-\text { a.s. }
$$

or equivalently (noticing $R>0$ ),

$$
\left\langle R^{\frac{1}{2}}\left[R^{-1}\left(B^{T} p^{i, *}+D^{T} q^{i, *}\right)-u^{i, *}\right], R^{\frac{1}{2}}\left(u-u^{i, *}\right)\right\rangle \leq 0, \text { for all } u \in \Gamma \text {, a.e. } t \in[0, T], \mathbb{P}-\text { a.s. }
$$

If we take the following norm on $\Gamma \subset \mathbb{R}^{m}$ (which is equivalent to its Euclidean norm)

optimal contr

$$
\|x\|_{R}^{2}=\langle\langle x, x\rangle\rangle:=\left\langle R^{\frac{1}{2}} x, R^{\frac{1}{2}} x\right\rangle,
$$

and by the well-known results of convex analysis, we obtain that (6) is equivalent to

$$
u^{i, *}(t)=\mathbf{P}_{\Gamma}\left[R^{-1}(t)\left(B^{T}(t) p^{i, *}(t)+D^{T}(t) q^{i, *}(t)\right)\right], \quad \text { a.e. } t \in[0, T], \mathbb{P}-\text { a.s. },
$$

where $\mathbf{P}_{\Gamma}(\cdot)$ is the projection mapping from $\mathbb{R}^{m}$ to its closed convex subset $\Gamma$ under the norm $\|\cdot\|_{R}$. For more details, see Appendix. From now on, we denote

$$
\varphi(t, p, q):=\mathbf{P}_{\Gamma}\left[R^{-1}(t)\left(B^{T}(t) p+D^{T}(t) q\right)\right] .
$$

Then the related Hamiltonian system becomes

$$
\left\{\begin{aligned}
d x^{i, *} & =\left[A x^{i, *}+B \varphi\left(p^{i, *}, q^{i, *}\right)+F z+b\right] d t+\left[D \varphi\left(p^{i, *}, q^{i, *}\right)+\sigma\right] d W_{i}(t), \\
d p^{i, *} & =-\left[A^{T} p^{i, *}-Q\left(x^{i, *}-z\right)\right] d t+q^{i, *} d W_{i}(t) \\
x^{i, *}(0) & =x, \quad p^{i, *}(T)=-G\left(x^{i, *}(T)-z(T)\right)
\end{aligned}\right.
$$

By the consistency condition, it follows that

$$
z(\cdot)=\lim _{N \rightarrow+\infty} \frac{1}{N} \sum_{i=1}^{N} x^{i, *}(\cdot)=\mathbb{E} x^{i, *}(\cdot) .
$$

limit average 
Thus, by replacing $z$ by $\mathbb{E} x^{i, *}$ in above, we get the following system

$$
\left\{\begin{aligned}
d x^{i, *} & =\left[A x^{i, *}+B \varphi\left(p^{i, *}, q^{i, *}\right)+F \mathbb{E} x^{i, *}+b\right] d t+\left[D \varphi\left(p^{i, *}, q^{i, *}\right)+\sigma\right] d W_{i}(t), \\
d p^{i, *} & =-\left[A^{T} p^{i, *}-Q\left(x^{i, *}-z\right)\right] d t+q^{i, *} d W_{i}(t), \\
x^{i, *}(0) & =x, \quad p^{i, *}(T)=-G\left(x^{i, *}(T)-z(T)\right) .
\end{aligned}\right.
$$

We have the following consistency condition system for generic agent (we suppress subscript here):

$$
\left\{\begin{aligned}
d x & =[A x+B \varphi(p, q)+F \mathbb{E} x+b] d t+[D \varphi(p, q)+\sigma] d W_{t}, \\
-d p & =\left[A^{T} p-Q(x-\mathbb{E} x)\right] d t-q d W_{t}, \\
x_{0} & =x, \quad p_{T}=-G\left(x_{T}-\mathbb{E} x_{T}\right) .
\end{aligned}\right.
$$

The above system is a nonlinear mean-field forward-backward SDE (MF-FBSDE) with projection operator. It characterizes the state-average limit $z=\mathbb{E} x$ and MFG strategies $\bar{u}_{i}=\varphi(p, q)$ for a generic agent in the combined manner. As an important issue, we need to prove the above consistency condition system admits a unique solution. We first present the following uniqueness and existence result.

Theorem 2.1 Under (H1), (H2), there exists a unique adapted solution $(x, p, q) \in L_{\mathbb{F} W}^{2}\left(0, T ; \mathbb{R}^{n}\right) \times$ $L_{\mathbb{F} W}^{2, \mathcal{E}_{0}}\left(0, T ; \mathbb{R}^{n}\right) \times L_{\mathbb{F} W}^{2}\left(0, T ; \mathbb{R}^{n}\right)$ to system (묘).

Proof (Uniqueness) Suppose that there exists two solutions: $\left(x^{1}, p^{1}, q^{1}\right),\left(x^{2}, p^{2}, q^{2}\right)$ and denote

$$
\hat{x}=x^{1}-x^{2}, \quad \hat{p}=p^{1}-p^{2}, \quad \hat{q}=q^{1}-q^{2} .
$$

Then, we have

$$
\left\{\begin{aligned}
d \hat{x} & =[A \hat{x}+B \widehat{\varphi}(\hat{p}, \hat{q})+F \mathbb{E} \hat{x}] d t+D \widehat{\varphi}(\hat{p}, \hat{q}) d W_{t} \\
-d \hat{p} & =\left[A^{T} \hat{p}-Q(\hat{x}-\mathbb{E} \hat{x})\right] d t-\hat{q} d W_{t} \\
\hat{x}_{0} & =0, \quad \hat{p}_{T}=-G\left(\hat{x}_{T}-\mathbb{E} \hat{x}_{T}\right)
\end{aligned}\right.
$$

with

$$
\widehat{\varphi}(\hat{p}, \hat{q}):=\varphi\left(p^{1}, q^{1}\right)-\varphi\left(p^{2}, q^{2}\right):=\mathbf{P}_{\Gamma}\left[R^{-1}\left(B^{T} p^{1}+D^{T} q^{1}\right)\right]-\mathbf{P}_{\Gamma}\left[R^{-1}\left(B^{T} p^{2}+D^{T} q^{2}\right)\right] .
$$

First, taking the expectation in the second equation of (9) yields $\mathbb{E} \hat{p}=0$. Applying Itô's formula to $\langle\hat{p}, \hat{x}\rangle$ and taking expectations on both sides:

$$
\begin{aligned}
0 & =\mathbb{E}\left\langle G\left(\hat{x}_{T}-\mathbb{E} \hat{x}_{T}\right), \hat{x}_{T}\right\rangle \\
& +\mathbb{E} \int_{0}^{T}\left\langle\left(B^{T} \hat{p}_{s}+D^{T} \hat{q}_{s}\right), \widehat{\varphi}_{s}(\hat{p}, \hat{q})\right\rangle+\left\langle\hat{x}_{s}, Q\left(\hat{x}_{s}-\mathbb{E} \hat{x}_{s}\right)\right\rangle d s+\mathbb{E} \int_{0}^{T}\left\langle\hat{p}_{s}, F \mathbb{E} \hat{x}_{s}\right\rangle d s \\
& \geq \mathbb{E}\left\langle G^{\frac{1}{2}}\left(\hat{x}_{T}-\mathbb{E} \hat{x}_{T}\right), G^{\frac{1}{2}}\left(\hat{x}_{T}-\mathbb{E} \hat{x}_{T}\right)\right\rangle+\mathbb{E} \int_{0}^{T}\left\langle Q^{\frac{1}{2}}\left(\hat{x}_{s}-\mathbb{E} \hat{x}_{s}\right), Q^{\frac{1}{2}}\left(\hat{x}_{s}-\mathbb{E} \hat{x}_{s}\right)\right\rangle d s .
\end{aligned}
$$

Thus, we have, $G\left(\hat{x}_{T}-\mathbb{E} \hat{x}_{T}\right)=0$ and $Q(\hat{x}-\mathbb{E} \hat{x})=0$ which implies $\hat{p}_{s} \equiv 0, \hat{q}_{s} \equiv 0$. Next, we have $\widehat{\varphi}_{s}(\hat{p}, \hat{q}) \equiv 0$ which further implies $\mathbb{E} \hat{x}_{s} \equiv 0$, hence $\hat{x}_{s} \equiv 0$. Hence the uniqueness follows.

(Existence) Consider a family of parameterized FBSDE as follows:

$$
\left\{\begin{aligned}
d x^{\alpha} & =\left[\alpha \mathbf{B}\left(x, p^{\alpha}, q^{\alpha}, \mathbb{E} x^{\alpha}\right)+\phi\right] d t+\left[\alpha \Xi\left(x, p^{\alpha}, q^{\alpha}, \mathbb{E} x^{\alpha}\right)+\psi\right] d W_{t}, \\
-d p^{\alpha} & =\left[\alpha \mathbf{F}\left(x^{\alpha}, p^{\alpha}, \mathbb{E} x^{\alpha}\right)+\gamma-\mathbb{E} \gamma\right] d t-q^{\alpha} d W_{t}, \\
x_{0}^{\alpha} & =x, \quad p_{T}^{\alpha}=-\alpha G\left(x_{T}^{\alpha}-\mathbb{E} x_{T}^{\alpha}\right)+\xi-\mathbb{E} \xi
\end{aligned}\right.
$$


with

$$
\left\{\begin{array}{l}
\mathbf{B}:=A x+B \varphi(p, q)+F_{1} \mathbb{E} x+b, \\
\Xi:=D \varphi(p, q)+\sigma, \\
\mathbf{F}:=A^{T} p-Q(x-\mathbb{E} x) .
\end{array}\right.
$$

Here $(\phi, \psi, \gamma)$ are given process in $L_{\mathbb{F}^{W}}^{2}\left(0, T ; \mathbb{R}^{n}\right) \times L_{\mathbb{F} W}^{2}\left(0, T ; \mathbb{R}^{n}\right) \times L_{\mathbb{F} W}^{2}\left(0, T ; \mathbb{R}^{n}\right)$, and $\xi$ is a $\mathbb{R}^{n}$-valued square integrable random variable which is $\mathbb{F}_{T}^{W}$-measurable. When $\alpha=0$, we have a decoupled FBSDE whose solvability is trivial:

$$
\left\{\begin{aligned}
d x & =\phi d t+\psi d W_{t}, \\
-d p & =(\gamma-\mathbb{E} \gamma) d t-q d W_{t}, \\
x_{0} & =x, \quad p_{T}=\xi-\mathbb{E} \xi
\end{aligned}\right.
$$

Denote $\mathcal{M}(0, T)=L_{\mathbb{F} W}^{2}\left(0, T ; \mathbb{R}^{n}\right) \times L_{\mathbb{F} W}^{2, \mathcal{E}_{0}}\left(0, T ; \mathbb{R}^{n}\right) \times L_{\mathbb{F} W}^{2}\left(0, T ; \mathbb{R}^{n}\right)$. Now introduce a mapping $I_{\alpha_{0}}:(x, p, q) \in \mathcal{M}(0, T) \longrightarrow(X, P, Q) \in \mathcal{M}(0, T)$ via the following FBSDE:

$$
\left\{\begin{aligned}
d X_{t} & =\left[\alpha_{0} \mathbf{B}\left(X_{t}, P_{t}, Q_{t}, \mathbb{E} X_{t}\right)+\delta \mathbf{B}\left(x_{t}, p_{t}, q_{t}, \mathbb{E} x_{t}\right)+\phi_{t}\right] d t \\
+ & {\left[\alpha_{0} \Xi\left(X_{t}, P_{t}, Q_{t}\right)+\delta \Xi\left(x_{t}, p_{t}, q_{t}\right)+\psi_{t}\right] d W_{t}, } \\
-d P_{t} & =\left[\alpha_{0} \mathbf{F}\left(X_{t}, P_{t}, Q_{t}, \mathbb{E} X_{t}\right)+\gamma_{t}-\mathbb{E} \gamma+\delta \mathbf{F}\left(x_{t}, p_{t}, q_{t}, \mathbb{E} x_{t}\right)\right] d t-Q_{t} d W_{t}, \\
X_{0} & =x, \quad P_{T}=-\alpha_{0} G\left(X_{T}-\mathbb{E} X_{T}\right)-\delta G\left(x_{T}-\mathbb{E} x_{T}\right)+\xi-\mathbb{E} \xi .
\end{aligned}\right.
$$

Considering $I_{\alpha_{0}}:(x, p, q) \longrightarrow(X, P, Q)$ and $I_{\alpha_{0}}:\left(x^{\prime}, p^{\prime}, q^{\prime}\right) \longrightarrow\left(X^{\prime}, P^{\prime}, Q^{\prime}\right)$ and

$$
\begin{gathered}
(\widehat{X}, \widehat{P}, \widehat{Q})=\left(X-X^{\prime}, P-P^{\prime}, Q-Q^{\prime}\right) \\
\left\{\begin{aligned}
d \widehat{X}_{t} & =\left[\alpha_{0} \widehat{\mathbf{B}}\left(\widehat{X}_{t}, \widehat{P}_{t}, \widehat{Q}_{t}, \mathbb{E} \widehat{X}_{t}\right)+\delta \widehat{\mathbf{B}}\left(\hat{x}_{t}, \hat{p}_{t}, \hat{q}_{t}, \mathbb{E} \hat{x}_{t}\right)\right] d t \\
+ & {\left[\alpha_{0} \widehat{\Xi}\left(\widehat{X}_{t}, \widehat{P}_{t}, \widehat{Q}_{t}\right)+\delta \widehat{\Xi}\left(\hat{x}_{t}, \hat{p}_{t}, \hat{q}_{t}\right)\right] d W_{t}, } \\
-d \widehat{P}_{t}= & {\left[\alpha_{0} \widehat{\mathbf{F}}\left(\widehat{X}_{t}, \widehat{P}_{t}, \widehat{Q}_{t}, \mathbb{E} \widehat{X}_{t}\right)+\delta\left(\widehat{\mathbf{F}}\left(\hat{x}_{t}, \hat{p}_{t}, \hat{q}_{t}, \mathbb{E} \hat{x}_{t}\right)\right] d t-\widehat{Q}_{t} d W_{t},\right.} \\
\widehat{X}_{0}= & 0, \quad \widehat{P}_{T}=-\alpha_{0} G\left(\widehat{X}_{T}-\mathbb{E} \widehat{X}_{T}\right)-\delta G\left(\hat{x}_{T}-\mathbb{E} \hat{x}_{T}\right),
\end{aligned}\right.
\end{gathered}
$$

with

$$
\left\{\begin{array}{l}
\widehat{\mathbf{B}}:=\mathbf{B}\left(X_{t}, P_{t}, Q_{t}, \mathbb{E} X_{t}\right)-\mathbf{B}\left(X_{t}^{\prime}, P_{t}^{\prime}, Q_{t}^{\prime}, \mathbb{E} X_{t}^{\prime}\right), \\
\widehat{\Xi}:=\Xi\left(X_{t}, P_{t}, Q_{t}, \mathbb{E} X_{t}\right)-\Xi\left(X_{t}^{\prime}, P_{t}^{\prime}, Q_{t}^{\prime}, \mathbb{E} X_{t}^{\prime}\right), \\
\widehat{\mathbf{F}}:=\mathbf{F}\left(X_{t}, P_{t}, Q_{t}, \mathbb{E} X_{t}\right)-\mathbf{F}\left(X_{t}^{\prime}, P_{t}^{\prime}, Q_{t}^{\prime}, \mathbb{E} X_{t}^{\prime}\right) .
\end{array}\right.
$$

Note that $\mathbb{E} \widehat{P}_{t} \equiv 0$ because

$$
\widehat{\mathbf{F}}\left(X_{t}, P_{t}, Q_{t}, \mathbb{E} X_{t}\right)=A^{T} \widehat{P}-Q(\widehat{X}-\mathbb{E} \widehat{X}), \quad \text { and } \quad \mathbb{E} p_{t} \equiv 0 .
$$

Applying Itô formula to $\langle\widehat{P}, \widehat{X}\rangle$ and taking expectations on both sides:

$$
\begin{aligned}
& \mathbb{E}\left\langle\widehat{X}_{T},-\alpha_{0} G\left(\widehat{X}_{T}-\mathbb{E} \widehat{X}_{T}\right)-\delta G\left(\hat{x}_{T}-\mathbb{E} \hat{x}_{T}\right)\right\rangle \\
& =\mathbb{E} \int_{0}^{T}\left\langle\widehat{X}_{s},-\alpha_{0} \widehat{\mathbf{F}}\left(\widehat{X}_{s}, \widehat{P}_{s}, \widehat{Q}_{s}, \mathbb{E} \widehat{X}_{s}\right)\right\rangle+\left\langle\widehat{X}_{s},-\delta \widehat{\mathbf{F}}\left(\hat{x}_{s}, \hat{p}_{s}, \hat{q}_{s}, \mathbb{E} \hat{x}_{s}\right)\right\rangle \\
& +\left\langle\widehat{P}_{s}, \alpha_{0} \widehat{\mathbf{B}}\left(\widehat{X}_{s}, \widehat{P}_{s}, \widehat{Q}_{s}, \mathbb{E} \widehat{X}_{s}\right)\right\rangle+\left\langle\widehat{P}_{s}, \delta \widehat{\mathbf{B}}\left(\hat{x}_{s}, \hat{p}_{s}, \hat{q}_{s}, \mathbb{E} \hat{x}_{s}\right)\right\rangle \\
& +\left\langle\widehat{Q}_{s}, \alpha_{0} \widehat{\Xi}\left(\widehat{X}_{s}, \widehat{P}_{s}, \widehat{Q}_{s}\right)\right\rangle+\left\langle\widehat{Q}_{s}, \delta \widehat{\mathbf{\Xi}}\left(\hat{x}_{s}, \hat{p}_{s}, \hat{q}_{s}\right)\right\rangle d s .
\end{aligned}
$$


Rearranging the above terms, we have

$$
\begin{aligned}
& \alpha_{0} \mathbb{E}\left\langle\widehat{X}_{T}, G\left(\widehat{X}_{T}-\mathbb{E} \widehat{X}_{T}\right)\right\rangle+\mathbb{E} \int_{0}^{T} \alpha_{0}\left[\left\langle\widehat{X}_{s},-\widehat{\mathbf{F}}\left(\widehat{X}_{s}, \widehat{P}_{s}, \widehat{Q}_{s}, \mathbb{E} \widehat{X}_{s}\right)\right\rangle\right. \\
& \left.+\left\langle\widehat{P}_{s}, \widehat{\mathbf{B}}\left(\widehat{X}_{s}, \widehat{P}_{s}, \widehat{Q}_{s}, \mathbb{E} \widehat{X}_{s}\right)\right\rangle+\left\langle\widehat{Q}_{s}, \widehat{\Xi}\left(\widehat{X}_{s}, \widehat{P}_{s}, \widehat{Q}_{s}\right)\right\rangle\right] d s \\
& =\mathbb{E} \int_{0}^{T} \delta\left[\left\langle\widehat{X}_{s}, \widehat{\mathbf{F}}\left(\hat{x}_{s}, \hat{p}_{s}, \hat{q}_{s}, \mathbb{E} \hat{x}_{s}\right)\right\rangle+\left\langle\widehat{P}_{s},-\widehat{\mathbf{B}}\left(\hat{x}_{s}, \hat{p}_{s}, \hat{q}_{s}, \mathbb{E} \hat{x}_{s}\right)\right\rangle\right. \\
& \left.+\left\langle\widehat{Q}_{s},-\widehat{\Xi}\left(\hat{x}_{s}, \hat{p}_{s}, \hat{q}_{s}\right)\right\rangle\right] d s-\delta \mathbb{E}\left\langle\widehat{X}_{T}, G\left(\hat{x}_{T}-\mathbb{E} \hat{x}_{T}\right)\right\rangle
\end{aligned}
$$

Hence,

$$
\begin{aligned}
& \alpha_{0} \mathbb{E}\left|G^{\frac{1}{2}}\left(\widehat{X}_{T}-\mathbb{E} \widehat{X}_{T}\right)\right|^{2}+\mathbb{E} \int_{0}^{T} \alpha_{0}\left|Q^{\frac{1}{2}}\left(\widehat{X}_{s}-\mathbb{E} \widehat{X}_{s}\right)\right|^{2} d s \\
& \leq \alpha_{0} \mathbb{E}\left\langle\widehat{X}_{T}, G\left(\widehat{X}_{T}-\mathbb{E} \widehat{X}_{T}\right)\right\rangle+\mathbb{E} \int_{0}^{T} \alpha_{0}\left[\left\langle\widehat{X}_{s},-\widehat{\mathbf{F}}\left(\widehat{X}_{s}, \widehat{P}_{s}, \widehat{Q}_{s}, \mathbb{E} \widehat{X}_{s}\right)\right\rangle\right. \\
& \left.+\left\langle\widehat{P}_{s}, \widehat{\mathbf{B}}\left(\widehat{X}_{s}, \widehat{P}_{s}, \widehat{Q}_{s}, \mathbb{E} \widehat{X}_{s}\right)\right\rangle+\left\langle\widehat{Q}_{s}, \widehat{\Xi}\left(\widehat{X}_{s}, \widehat{P}_{s}, \widehat{Q}_{s}\right)\right\rangle\right] d s \\
& =\mathbb{E} \int_{0}^{T} \delta\left[\left\langle\widehat{X}_{s}, \widehat{\mathbf{F}}\left(\hat{x}_{s}, \hat{p}_{s}, \hat{q}_{s}, \mathbb{E} \hat{x}_{s}\right)\right\rangle+\left\langle\widehat{P}_{s},-\widehat{\mathbf{B}}\left(\hat{x}_{s}, \hat{p}_{s}, \hat{q}_{s}, \mathbb{E} \hat{x}_{s}\right)\right\rangle\right. \\
& \left.+\left\langle\widehat{Q}_{s},-\widehat{\Xi}\left(\hat{x}_{s}, \hat{p}_{s}, \hat{q}_{s}\right)\right\rangle\right] d s-\delta \mathbb{E}\left\langle\widehat{X}_{T}, G\left(\hat{x}_{T}-\mathbb{E} \hat{x}_{T}\right)\right\rangle \\
& \leq \delta C_{1} \mathbb{E} \int_{0}^{T}\left(\left|\hat{x}_{s}\right|^{2}+\left|\hat{p}_{s}\right|^{2}+\left|\hat{q}_{s}\right|^{2}\right) d s+\delta C_{1} \mathbb{E} \hat{x}_{T}^{2}+\delta C_{1} \mathbb{E} \int_{0}^{T}\left(\left|\widehat{X}_{s}\right|^{2}+\left|\widehat{P}_{s}\right|^{2}+\left|\widehat{Q}_{s}\right|^{2}\right) d s+\delta C_{1} \mathbb{E} \widehat{X}_{T}^{2} .
\end{aligned}
$$

Then, by standard estimates of BSDE:

$$
\begin{aligned}
& \mathbb{E} \int_{0}^{T}\left(\left|\widehat{P}_{s}\right|^{2}+\left|\widehat{Q}_{s}\right|^{2}\right) d s \\
& \leq \delta C_{2} \mathbb{E} \int_{0}^{T}\left(\left|\hat{x}_{s}\right|^{2}+\left|\hat{p}_{s}\right|^{2}+\left|\hat{q}_{s}\right|^{2}\right) d s+\delta C_{2} \mathbb{E}\left|\hat{x}_{T}\right|^{2} \\
& +C_{2}\left(\alpha_{0} \mathbb{E}\left|G^{\frac{1}{2}}\left(\widehat{X}_{T}-\mathbb{E} \widehat{X}_{T}\right)\right|^{2}+\mathbb{E} \int_{0}^{T} \alpha_{0}\left|Q^{\frac{1}{2}}\left(\widehat{X}_{s}-\mathbb{E} \widehat{X}_{s}\right)\right|^{2} d s\right) \\
& \leq \delta C_{3} \mathbb{E} \int_{0}^{T}\left(\left|\hat{x}_{s}\right|^{2}+\left|\hat{p}_{s}\right|^{2}+\left|\hat{q}_{s}\right|^{2}\right) d s+\delta C_{3} \mathbb{E}\left|\hat{x}_{T}\right|^{2} .
\end{aligned}
$$

Next, by the standard estimate of forward SDEs:

$$
\begin{aligned}
& \mathbb{E} \int_{0}^{T}\left|\widehat{X}_{s}\right|^{2} d s+\mathbb{E}\left|\widehat{X}_{T}\right|^{2} \\
& \leq \delta C_{4} \mathbb{E} \int_{0}^{T}\left(\left|\hat{x}_{s}\right|^{2}+\left|\hat{p}_{s}\right|^{2}+\left|\hat{q}_{s}\right|^{2}\right) d s+C_{4} \mathbb{E} \int_{0}^{T}\left(\left|\widehat{P}_{s}\right|^{2}+\left|\widehat{Q}_{s}\right|^{2}\right) d s \\
& \leq \delta C_{5} \delta\left(\mathbb{E} \int_{0}^{T}\left(\left|\hat{x}_{s}\right|^{2}+\left|\hat{p}_{s}\right|^{2}+\left|\hat{q}_{s}\right|^{2}\right) d s+\delta C_{5} \mathbb{E}\left|\hat{x}_{T}\right|^{2}\right.
\end{aligned}
$$

Based on the above estimates, we know the mapping $I$ satisfying

$$
\mathbb{E} \int_{0}^{T}\left(\left|\widehat{X}_{s}\right|^{2}+\left|\widehat{P}_{s}\right|^{2}+\left|\widehat{Q}_{s}\right|^{2}\right) d s+\mathbb{E}\left|\widehat{X}_{T}\right|^{2} \leq K \delta\left(\mathbb{E} \int_{0}^{T}\left(\left|\widehat{x}_{s}\right|^{2}+\left|\widehat{p}_{s}\right|^{2}+\left|\widehat{q}_{s}\right|^{2}\right) d s+\mathbb{E}\left|\widehat{x}_{T}\right|^{2}\right) .
$$

It follows the mapping is a contraction and the existence follows immediately using the arguments presented in [8] and [15]. 


\section{$3 \epsilon$-Nash Equilibrium for Problem (CC)}

In above sections, we can characterize the decentralized strategies $\left\{\bar{u}_{t}^{i}, 1 \leq i \leq N\right\}$ of Problem (CC) through the auxiliary (LCC) and consistency condition system. For sake of presentation, we alter the notations of consistency condition system to be $\left(\alpha^{i}, \beta^{i}, \gamma^{i}\right)$ :

$$
\left\{\begin{aligned}
d \alpha^{i} & =\left[A \alpha^{i}+B \varphi\left(\beta^{i}, \gamma^{i}\right)+F \mathbb{E} \alpha^{i}+b\right] d t+\left[D \varphi\left(\beta^{i}, \gamma^{i}\right)+\sigma\right] d W_{i}(t), \\
d \beta^{i} & =-\left(A^{T} \beta^{i}-Q\left(\alpha^{i}-\mathbb{E} \alpha^{i}\right)\right) d t+\gamma^{i} d W_{i}(t) \\
\alpha^{i}(0) & =x, \quad \beta^{i}(T)=-G\left(\alpha^{i}(T)-\mathbb{E} \alpha^{i}(T)\right) .
\end{aligned}\right.
$$

Now, we turn to verify the $\epsilon$-Nash equilibrium of them. To start, we first present the definition of $\epsilon$-Nash equilibrium.

d1 Definition 3.1 A set of strategies $\bar{u}_{t}^{i} \in \mathcal{U}_{a d}^{c}, 1 \leq i \leq N$, for $N$ agents is called to satisfy an $\epsilon$-Nash equilibrium with respect to costs $\mathcal{J}^{i}, 1 \leq i \leq N$, if there exists $\epsilon \geq 0$ such that for any fixed $1 \leq i \leq N$, we have

$$
\mathcal{J}^{i}\left(\bar{u}_{t}^{i}, \bar{u}_{t}^{-i}\right) \leq \mathcal{J}^{i}\left(u_{t}^{i}, \bar{u}_{t}^{-i}\right)+\epsilon,
$$

when any alternative strategy $u^{i} \in \mathcal{U}_{\text {ad }}^{c}$ is applied by $\mathcal{A}_{i}$.

Remark 3.1 If $\epsilon=0$, then Definition 3.1 is reduced to the usual exact Nash equilibrium.

Now, we state the main result of this paper and its proof will be given later.

Theorem 3.1 Under (H1)-(H2), $\left(\bar{u}_{1}, \bar{u}_{2}, \cdots, \bar{u}_{N}\right)$ is an $\epsilon$-Nash equilibrium of Problem (CC).

The proof of Theorem 3.1 needs several lemmas which are presented later. For agent $\mathcal{A}_{i}$, recall that its decentralized open-loop optimal strategy is $\bar{u}_{i}=\varphi\left(\beta^{i}, \gamma^{i}\right)$. The decentralized state $\breve{x}_{t}^{i}$ is

$$
\left\{\begin{aligned}
d \breve{x}^{i} & =\left[A \breve{x}^{i}+B \varphi\left(\beta^{i}, \gamma^{i}\right)+F \breve{x}^{(N)}+b\right] d t+\left[D \varphi\left(\beta^{i}, \gamma^{i}\right)+\sigma\right] d W_{i}(t), \\
d \alpha^{i} & =\left[A \alpha^{i}+B \varphi\left(\beta^{i}, \gamma^{i}\right)+F \mathbb{E} \alpha^{i}+b\right] d t+\left[D \varphi\left(\beta^{i}, \gamma^{i}\right)+\sigma\right] d W_{i}(t), \\
d \beta^{i} & =-\left[A^{T} \beta^{i}-Q\left(\alpha^{i}-\mathbb{E} \alpha^{i}\right)\right] d t+\gamma^{i} d W_{i}(t), \\
\breve{x}^{i}(0) & =\alpha^{i}(0)=x, \quad \beta^{i}(T)=-G\left(\alpha^{i}(T)-\mathbb{E} \alpha^{i}(T)\right),
\end{aligned}\right.
$$

where $\breve{x}^{(N)}=\frac{1}{N} \sum_{i=1}^{N} \breve{x}^{i}$. For comparison, we prefer to write the limiting state $\alpha_{t}^{i}$ once again,

$$
\left\{\begin{aligned}
d \alpha^{i} & =\left[A \alpha^{i}+B \varphi\left(\beta^{i}, \gamma^{i}\right)+F \mathbb{E} \alpha^{i}+b\right] d t+\left[D \varphi\left(\beta^{i}, \gamma^{i}\right)+\sigma\right] d W_{i}(t), \\
d \beta^{i} & =-\left[A^{T} \beta^{i}-Q\left(\alpha^{i}-\mathbb{E} \alpha^{i}\right)\right] d t+\gamma^{i} d W_{i}(t), \\
\alpha^{i}(0) & =x, \quad \beta^{i}(T)=-G\left(\alpha^{i}(T)-\mathbb{E} \alpha^{i}(T)\right) .
\end{aligned}\right.
$$

Now, let us present the following lemmas.

Lemma 3.1 There exists a constant $C_{0}$ independent of $N$, such that

$$
\sup _{1 \leq i \leq N} \mathbb{E} \sup _{0 \leq t \leq T}\left|\breve{x}^{i}(t)\right|^{2} \leq C_{0} .
$$

Proof For each $1 \leq i \leq N$, the monotonic fully coupled FBSDE

$$
\left\{\begin{aligned}
d \alpha^{i} & =\left[A \alpha^{i}+B \varphi\left(\beta^{i}, \gamma^{i}\right)+F \mathbb{E} \alpha^{i}+b\right] d t+\left[D \varphi\left(\beta^{i}, \gamma^{i}\right)+\sigma\right] d W_{i}(t), \\
d \beta^{i} & =-\left[A^{T} \beta^{i}-Q\left(\alpha^{i}-\mathbb{E} \alpha^{i}\right)\right] d t+\gamma^{i} d W_{i}(t), \\
\alpha^{i}(0) & =x, \quad \beta^{i}(T)=-G\left(\alpha^{i}(T)-\mathbb{E} \alpha^{i}(T)\right),
\end{aligned}\right.
$$


has a unique solution $\left(\alpha^{i}, \beta^{i}, \gamma^{i}\right) \in L_{\mathbb{F}^{i}}^{2}\left(0, T ; \mathbb{R}^{n}\right) \times L_{\mathbb{F}^{i}}^{2}\left(0, T ; \mathbb{R}^{n}\right) \times L_{\mathbb{F}^{i}}^{2}\left(0, T ; \mathbb{R}^{n}\right)$. Thus, the system of all first equation of (10), $1 \leq i \leq N$, has also a unique solution $\left(\breve{x}^{i}\right)_{i} \in\left(L_{\mathbb{F}^{W} W_{1}, \cdots, W_{N}}^{2}\left(0, T ; \mathbb{R}^{n}\right)\right)^{\otimes N}$. Moreover, since $\left\{W_{i}\right\}_{i=1}^{N}$ is $N$-dimensional Brownian motion whose components are independent and identically distributed, we have $\left(\alpha^{i}, \beta^{i}, \gamma^{i}\right), 1 \leq i \leq N$ are independent identically distributed.

Noticing that $\left(\beta^{i}, \gamma^{i}\right) \in L_{\mathbb{F} i}^{2}\left(0, T ; \mathbb{R}^{n}\right) \times L_{\mathbb{F} i}^{2}\left(0, T ; \mathbb{R}^{n}\right)$, with the Lipschitz property of the projection onto convex set, it is not hard to show that $\varphi\left(\beta^{i}, \gamma^{i}\right):=\mathbf{P}_{\Gamma}\left(R^{-1}\left(B^{T} \beta^{i}+D^{T} \gamma^{i}\right)\right) \in$ $L_{\mathbb{F}^{i}}^{2}(0, T ; \Gamma)$. From the above analysis and the classical estimates of FBSDEs, we have that there exists a constant $C_{0}$ independent of $N$ which may very line by line in the following, such that

$$
\mathbb{E} \sup _{0 \leq t \leq T}\left(\left|\alpha^{i}(t)\right|^{2}+\left|\beta^{i}(t)\right|^{2}\right)+\mathbb{E} \int_{0}^{T}\left(\left|\gamma^{i}(t)\right|^{2}+\left|\varphi\left(\beta^{i}(t), \gamma^{i}(t)\right)\right|^{2}\right) d t \leq C_{0}
$$

Then from the first equation of (10), by using Burkholder-Davis-Gundy (BDG) inequality, we have, for any $t \in[0, T]$,

$$
\begin{aligned}
\mathbb{E} \sup _{0 \leq s \leq t}\left|\breve{x}^{i}(s)\right|^{2} & \leq C_{0}+C_{0} \mathbb{E} \int_{0}^{t}\left[\left|\breve{x}^{i}(s)\right|^{2}+\left|\breve{x}^{(N)}(s)\right|^{2}\right] d s \\
& \leq C_{0}+C_{0} \mathbb{E} \int_{0}^{t}\left[\left|\breve{x}^{i}(s)\right|^{2}+\frac{1}{N} \sum_{i=1}^{N}\left|\breve{x}^{i}(s)\right|^{2}\right] d s .
\end{aligned}
$$

Thus

$$
\mathbb{E} \sup _{0 \leq s \leq t} \sum_{i=1}^{N}\left|\breve{x}^{i}(s)\right|^{2} \leq \mathbb{E} \sum_{i=1}^{N} \sup _{0 \leq s \leq t}\left|\breve{x}^{i}(s)\right|^{2} \leq C_{0} N+2 C_{0} \mathbb{E} \int_{0}^{t}\left[\sum_{i=1}^{N}\left|\breve{x}^{i}(s)\right|^{2}\right] d s .
$$

By Gronwall's inequality, it is easy to obtain

$$
\mathbb{E} \sup _{0 \leq s \leq t} \sum_{i=1}^{N}\left|\breve{x}^{i}(s)\right|^{2}=O(N), \text { for any } 1 \leq i \leq N .
$$

Finally, substituting this estimate to (12) and using Gronwall's inequality once again, we have

$$
\mathbb{E} \sup _{0 \leq t \leq T}\left|\breve{x}^{i}(t)\right|^{2} \leq C_{0}, \text { for any } 1 \leq i \leq N
$$

which completes the proof.

Lemma 3.2

$$
\mathbb{E} \sup _{0 \leq t \leq T}\left|\breve{x}^{(N)}(t)-\mathbb{E} \alpha^{i}(t)\right|^{2}=O\left(\frac{1}{N}\right)
$$

Proof On one hand, let us add up both sides of the first equation of (10) with respect to all $1 \leq i \leq N$ and multiply $\frac{1}{N}$, we obtain (recall that $\breve{x}^{(N)}=\frac{1}{N} \sum_{i=1}^{N} \breve{x}^{i}$ )

$$
\left\{\begin{aligned}
d \breve{x}^{(N)}= & {\left[A \breve{x}^{(N)}+\frac{1}{N} \sum_{i=1}^{N} B \varphi\left(\beta^{i}, \gamma^{i}\right)+F \breve{x}^{(N)}+b\right] d t } \\
& +\frac{1}{N} \sum_{i=1}^{N}\left[D \varphi\left(\beta^{i}, \gamma^{i}\right)+\sigma\right] d W_{i}(t), \\
\breve{x}^{(N)}(0)= & x .
\end{aligned}\right.
$$


On the other hand, by taking the expectation on both sides of the second equation of (10), it follows from Fubini's theorem that $\mathbb{E} \alpha^{i}$ satisfies the following equation:

$$
\left\{\begin{array}{l}
d\left(\mathbb{E} \alpha^{i}\right)=\left[A \mathbb{E} \alpha^{i}+\mathbb{E}\left(B \varphi\left(\beta^{i}, \gamma^{i}\right)\right)+F \mathbb{E} \alpha^{i}+b\right] d t, \\
\mathbb{E} \alpha^{i}(0)=x
\end{array}\right.
$$

From (14) and (15), by denoting $\Delta(t):=\breve{x}^{(N)}(t)-\mathbb{E} \alpha^{i}(t)$, we have

$$
\left\{\begin{aligned}
d \Delta= & {\left[A \Delta+\frac{1}{N} \sum_{i=1}^{N} B \varphi\left(\beta^{i}, \gamma^{i}\right)-B \mathbb{E} \varphi\left(\beta^{i}, \gamma^{i}\right)+F \Delta\right] d t } \\
& +\frac{1}{N} \sum_{i=1}^{N}\left[D \varphi\left(\beta^{i}, \gamma^{i}\right)+\sigma\right] d W_{i}(t), \\
\Delta(0)= & 0,
\end{aligned}\right.
$$

and the inequality $(x+y)^{2} \leq 2 x^{2}+2 y^{2}$ yields that, for any $t \in[0, T]$,

$$
\begin{aligned}
\mathbb{E} \sup _{0 \leq s \leq t}|\Delta(s)|^{2} & \leq 2 \mathbb{E} \sup _{0 \leq s \leq t}\left|\int_{0}^{s}\left[(A+F) \Delta(r)+\frac{1}{N} \sum_{i=1}^{N} B \varphi\left(\beta^{i}(r), \gamma^{i}(r)\right)-B \mathbb{E} \varphi\left(\beta^{i}(r), \gamma^{i}(r)\right)\right] d r\right|^{2} \\
& +2 \mathbb{E} \sup _{0 \leq s \leq t}\left|\frac{1}{N} \sum_{i=1}^{N} \int_{0}^{s}\left[D \varphi\left(\beta^{i}(r), \gamma^{i}(r)\right)+\sigma(r)\right] d W_{i}(r)\right|^{2} .
\end{aligned}
$$

From the Cauchy-Schwartz inequality and the BDG inequality, we obtain that there exists a constant $C_{0}$ independent of $N$ (which may vary line by line) such that

$$
\begin{aligned}
\mathbb{E} \sup _{0 \leq s \leq t}|\Delta(t)|^{2} \leq C_{0} \mathbb{E} \int_{0}^{t}\left[|\Delta(s)|^{2}+\left|\frac{1}{N} \sum_{i=1}^{N} \varphi\left(\beta^{i}(s), \gamma^{i}(s)\right)-\mathbb{E} \varphi\left(\beta^{i}(s), \gamma^{i}(s)\right)\right|^{2}\right] d s \\
+\frac{C_{0}}{N^{2}} \mathbb{E}\left(\sum_{i=1}^{N} \int_{0}^{t}\left|D \varphi\left(\beta^{i}(s), \gamma^{i}(s)\right)+\sigma(s)\right|^{2} d s\right) .
\end{aligned}
$$

Since $\left(\beta^{i}, \gamma^{i}\right), 1 \leq i \leq N$ are independent identically distributed, for each fixed $s \in[0, T]$, let us denote that $\mu(s)=\mathbb{E} \varphi\left(\beta^{i}(s), \gamma^{i}(s)\right.$ ) (note that $\mu$ does not depend on $i$ ), we have

$$
\begin{aligned}
& \mathbb{E}\left|\frac{1}{N} \sum_{i=1}^{N} \varphi\left(\beta^{i}(s), \gamma^{i}(s)\right)-\mu(s)\right|^{2}=\frac{1}{N^{2}} \mathbb{E}\left|\sum_{i=1}^{N}\left[\varphi\left(\beta^{i}(s), \gamma^{i}(s)\right)-\mu(s)\right]\right|^{2} \\
= & \frac{1}{N^{2}} \mathbb{E} \sum_{i=1}^{N}\left|\varphi\left(\beta^{i}(s), \gamma^{i}(s)\right)-\mu(s)\right|^{2} \\
& +\frac{1}{N^{2}} \mathbb{E} \sum_{i=1, j=1, j \neq i,}^{N}\left\langle\varphi\left(\beta^{i}(s), \gamma^{i}(s)\right)-\mu(s), \varphi\left(\beta^{j}(s), \gamma^{j}(s)\right)-\mu(s)\right\rangle .
\end{aligned}
$$

Since $\left(\beta^{i}, \gamma^{i}\right), 1 \leq i \leq N$ are independent, we have

$$
\begin{aligned}
& \frac{1}{N^{2}} \mathbb{E} \sum_{i=1, j=1, j \neq i,}^{N}\left\langle\varphi\left(\beta^{i}(s), \gamma^{i}(s)\right)-\mu(s), \varphi\left(\beta^{j}(s), \gamma^{j}(s)\right)-\mu(s)\right\rangle \\
= & \frac{1}{N^{2}} \sum_{i=1, j=1, j \neq i,}^{N}\left\langle\mathbb{E} \varphi\left(\beta^{i}(s), \gamma^{i}(s)\right)-\mu(s), \mathbb{E} \varphi\left(\beta^{j}(s), \gamma^{j}(s)\right)-\mu(s)\right\rangle=0 .
\end{aligned}
$$


Then, due to the fact that $\left(\beta^{i}, \gamma^{i}\right), 1 \leq i \leq N$ are identically distributed, we can obtain that there exists a constant $C_{0}$ independent of $N$ such that

$$
\begin{aligned}
& \int_{0}^{t} \mathbb{E}\left|\frac{1}{N} \sum_{i=1}^{N} B \varphi\left(\beta^{i}(s), \gamma^{i}(s)\right)-B \mathbb{E} \varphi\left(\beta^{i}(s), \gamma^{i}(s)\right)\right|^{2} d s \\
\leq & C_{0} \int_{0}^{t} \mathbb{E}\left|\frac{1}{N} \sum_{i=1}^{N} \varphi\left(\beta^{i}(s), \gamma^{i}(s)\right)-\mu(s)\right|^{2} d s=\frac{C_{0}}{N^{2}} \int_{0}^{t} \mathbb{E} \sum_{i=1}^{N}\left|\varphi\left(\beta^{i}(s), \gamma^{i}(s)\right)-\mu(s)\right|^{2} d s \\
= & \frac{C_{0}}{N} \int_{0}^{t} \mathbb{E}\left|\varphi\left(\beta^{i}(s), \gamma^{i}(s)\right)-\mu(s)\right|^{2} d s=O\left(\frac{1}{N}\right),
\end{aligned}
$$

where the last equality comes from the fact that $\varphi\left(\beta^{i}, \gamma^{i}\right) \in L_{\mathcal{F}^{i}}^{2}(0, T ; \Gamma)$.

Let us now estimate the second term of (16), using the fact that $\left(\alpha^{i}, \beta^{i}, \gamma^{i}\right)$ are identically distributed, we have

$$
\frac{C_{0}}{N^{2}} \mathbb{E}\left(\sum_{i=1}^{N} \int_{0}^{t}\left|D \varphi\left(\beta^{i}(s), \gamma^{i}(s)\right)+\sigma(s)\right|^{2} d s\right)=O\left(\frac{1}{N}\right) .
$$

Therefore, from the above analysis, we get from (16) that

$$
\mathbb{E} \sup _{0 \leq s \leq t}|\Delta(s)|^{2} \leq C_{0} \mathbb{E} \int_{0}^{t}|\Delta(s)|^{2}+O\left(\frac{1}{N}\right), \text { for any } t \in[0, T] .
$$

Finally, by using Gronwall's inequality, we complete the proof.

\section{Lemma 3.3}

$$
\sup _{1 \leq i \leq N} \mathbb{E} \sup _{0 \leq t \leq T}\left|\breve{x}^{i}(t)-\alpha^{i}(t)\right|^{2}=O\left(\frac{1}{N}\right) .
$$

estimate for

Proof From (10) and (11), we have that

$$
\left\{\begin{aligned}
d \breve{x}^{i} & =\left[A \breve{x}^{i}+B \varphi\left(\beta^{i}, \gamma^{i}\right)+F \breve{x}^{(N)}+b\right] d t+\left[D \varphi\left(\beta^{i}, \gamma^{i}\right)+\sigma\right] d W_{i}(t), \\
d \alpha^{i} & =\left[A \alpha^{i}+B \varphi\left(\beta^{i}, \gamma^{i}\right)+F \mathbb{E} \alpha^{i}+b\right] d t+\left[D \varphi\left(\beta^{i}, \gamma^{i}\right)+\sigma\right] d W_{i}(t), \\
\breve{x}^{i}(0) & =\alpha^{i}(0)=x
\end{aligned}\right.
$$

where $\left(\alpha^{i}, \beta^{i}, \gamma^{i}\right)$ is the unique solution to the following FBSDE:

$$
\left\{\begin{aligned}
d \alpha^{i} & =\left[A \alpha^{i}+B \varphi\left(\beta^{i}, \gamma^{i}\right)+F \mathbb{E} \alpha^{i}+b\right] d t+\left[D \varphi\left(\beta^{i}, \gamma^{i}\right)+\sigma\right] d W_{i}(t), \\
d \beta^{i} & =-\left[A^{T} \beta^{i}-Q\left(\alpha^{i}-\mathbb{E} \alpha^{i}\right)\right] d t+\gamma^{i} d W_{i}(t), \\
\alpha^{i}(0) & =x, \quad \beta^{i}(T)=-G\left(\alpha_{T}^{i}-\mathbb{E} \alpha_{T}^{i}\right) .
\end{aligned}\right.
$$

From (18), we have

$$
\left\{\begin{aligned}
d\left(\breve{x}^{i}-\alpha^{i}\right) & =\left[A\left(\breve{x}^{i}-\alpha^{i}\right)+F\left(\breve{x}^{(N)}-\mathbb{E} \alpha^{i}\right)\right] d t, \\
\breve{x}^{i}(0)-\bar{x}^{i}(0) & =0 .
\end{aligned}\right.
$$

The classical estimate for the SDE yields that

$$
\mathbb{E} \sup _{0 \leq t \leq T}\left|\breve{x}^{i}(t)-\alpha^{i}(t)\right|^{2} \leq C_{0} \mathbb{E} \int_{0}^{T}\left|\breve{x}^{(N)}(s)-\mathbb{E} \alpha^{i}(s)\right|^{2} d s,
$$

where $C_{0}$ is a constant independent of $N$. Noticing (13) of Lemma 3.2, we obtain (17). The proof is completed. 
Lemma for cost 3.4 For all $1 \leq i \leq N$, we have

$$
\left|\mathcal{J}_{i}\left(\bar{u}_{i}, \bar{u}_{-i}\right)-J_{i}\left(\bar{u}_{i}\right)\right|=O\left(\frac{1}{\sqrt{N}}\right)
$$

Proof Recall (2), (3) and (7), we have

$$
\begin{aligned}
\mathcal{J}_{i}\left(\bar{u}_{i}, \bar{u}_{-i}\right) & =\frac{1}{2} \mathbb{E}\left[\int_{0}^{T}\left\langle Q(t)\left(\breve{x}^{i}(t)-\breve{x}^{(N)}(t)\right), \breve{x}^{i}(t)-\breve{x}^{(N)}(t)\right\rangle\right. \\
& \left.+\left\langle R(t) \bar{u}_{i}(t), \bar{u}_{i}(t)\right\rangle d t+\left\langle G\left(\breve{x}^{i}(T)-\breve{x}^{(N)}(T)\right), \breve{x}^{i}(T)-\breve{x}^{(N)}(T)\right\rangle\right]
\end{aligned}
$$

and

$$
\begin{aligned}
J_{i}\left(\bar{u}_{i}\right) & =\frac{1}{2} \mathbb{E}\left[\int_{0}^{T}\left\langle Q(t)\left(\alpha^{i}(t)-\mathbb{E} \alpha^{i}(t)\right), \alpha^{i}(t)-\mathbb{E} \alpha^{i}(t)\right\rangle d t\right. \\
& \left.+\left\langle R(t) \bar{u}_{i}(t), \bar{u}_{i}(t)\right\rangle d t+\left\langle G\left(\alpha^{i}(T)-\mathbb{E} \alpha^{i}(T)\right), \alpha^{i}(T)-\mathbb{E} \alpha^{i}(T)\right\rangle\right]
\end{aligned}
$$

then

$$
\begin{aligned}
& \mathcal{J}_{i}\left(\bar{u}_{i}, \bar{u}_{-i}\right)-J_{i}\left(\bar{u}_{i}\right) \\
= & \frac{1}{2} \mathbb{E}\left[\int_{0}^{T}\left(\left\langle Q(t)\left(\breve{x}^{i}(t)-\breve{x}^{(N)}(t)\right), \breve{x}^{i}(t)-\breve{x}^{(N)}(t)\right\rangle-\left\langle Q(t)\left(\alpha^{i}(t)-\mathbb{E} \alpha^{i}(t)\right), \alpha^{i}(t)-\mathbb{E} \alpha^{i}(t)\right\rangle\right) d t\right. \\
& \left.+\left\langle G\left(\breve{x}^{i}(T)-\breve{x}^{(N)}(T)\right), \breve{x}^{i}(T)-\breve{x}^{(N)}(T)\right\rangle-\left\langle G\left(\alpha^{i}(T)-\mathbb{E} \alpha^{i}(T)\right), \alpha^{i}(T)-\mathbb{E} \alpha^{i}(T)\right\rangle\right] .
\end{aligned}
$$

From

$$
\begin{aligned}
& \langle Q(a-b), a-b\rangle-\langle Q(c-d), c-d\rangle \\
= & \langle Q(a-b-(c-d)), a-b-(c-d)\rangle+2\langle Q(a-b-(c-d)), c-d\rangle,
\end{aligned}
$$

and Lemma 3.2. Lemma 3.3 as well as $\mathbb{E} \sup _{0 \leq t \leq T}\left|\alpha^{i}(t)\right|^{2} \leq C_{0}$, for some constant $C_{0}$ independent of $N$ which may vary line by line in the following, we have

$$
\begin{aligned}
& \mid \mathbb{E}\left[\int_{0}^{T}\left(\left\langle Q(t)\left(\breve{x}^{i}(t)-\breve{x}^{(N)}(t)\right), \breve{x}^{i}(t)-\breve{x}^{(N)}(t)\right\rangle-\left\langle Q(t)\left(\alpha^{i}(t)-\mathbb{E} \alpha^{i}(t)\right), \alpha^{i}(t)-\mathbb{E} \alpha^{i}(t)\right\rangle\right) d t \mid\right. \\
& \leq C_{0} \int_{0}^{T} \mathbb{E}\left|\breve{x}^{i}(t)-\breve{x}^{(N)}(t)-\left(\alpha^{i}(t)-\mathbb{E} \alpha^{i}(t)\right)\right|^{2} d t \\
& +C_{0} \int_{0}^{T} \mathbb{E}\left[\left|\breve{x}^{i}(t)-\breve{x}^{(N)}(t)-\left(\alpha^{i}(t)-\mathbb{E} \alpha^{i}(t)\right)\right| \cdot\left|\alpha^{i}(t)-\mathbb{E} \alpha^{i}(t)\right|\right] d t \\
& \leq C_{0} \int_{0}^{T} \mathbb{E}\left|\breve{x}^{i}(t)-\alpha^{i}(t)\right|^{2} d t+C_{0} \int_{0}^{T} \mathbb{E}\left|\breve{x}^{(N)}(t)-\mathbb{E} \alpha^{i}(t)\right|^{2} d t \\
& +C_{0} \int_{0}^{T}\left(\mathbb{E}\left|\breve{x}^{i}(t)-\breve{x}^{(N)}(t)-\left(\alpha^{i}(t)-\mathbb{E} \alpha^{i}(t)\right)\right|^{2}\right)^{\frac{1}{2}}\left(\mathbb{E}\left|\alpha^{i}(t)-\mathbb{E} \alpha^{i}(t)\right|^{2}\right)^{\frac{1}{2}} d t \\
& \leq C_{0} \int_{0}^{T} \mathbb{E}\left|\breve{x}^{i}(t)-\alpha^{i}(t)\right|^{2} d t+C_{0} \int_{0}^{T} \mathbb{E}\left|\breve{x}^{(N)}(t)-\mathbb{E} \alpha^{i}(t)\right|^{2} d t \\
& +C_{0} \int_{0}^{T}\left(\mathbb{E}\left|\breve{x}^{i}(t)-\alpha^{i}(t)\right|^{2}+\mathbb{E}\left|\breve{x}^{(N)}(t)-\mathbb{E} \alpha^{i}(t)\right|^{2}\right)^{\frac{1}{2}} d t \\
& =O\left(\frac{1}{\sqrt{N}}\right) \text {. }
\end{aligned}
$$


With similar argument, we can show that

$$
\left|\mathbb{E}\left[\left\langle G\left(\breve{x}^{i}(T)-\breve{x}^{(N)}(T)\right), \breve{x}^{i}(T)-\breve{x}^{(N)}(T)\right\rangle-\left\langle G\left(\alpha^{i}(T)-\mathbb{E} \alpha^{i}(T)\right), \alpha^{i}(T)-\mathbb{E} \alpha^{i}(T)\right\rangle\right]\right|=O\left(\frac{1}{\sqrt{N}}\right) .
$$

The proof is completed by noticing (19).

Our remaining analysis is to prove the control strategies set $\left(\bar{u}_{1}, \bar{u}_{2}, \ldots, \bar{u}_{N}\right)$ is an $\epsilon$-Nash equilibrium for Problem (CC). For any fixed $i, 1 \leq i \leq N$, we consider the perturbation control $u_{i} \in \mathcal{U}_{a d}^{d, i}$ and we have the following state dynamics $(j \neq i)$ :

$$
\left\{\begin{aligned}
d y^{i} & =\left[A y^{i}+B u_{i}+F y^{(N)}+b\right] d t+\left[D u_{i}+\sigma\right] d W_{i}(t), \\
d y^{j} & =\left[A y^{j}+B \varphi\left(\beta^{j}, \gamma^{j}\right)+F y^{(N)}+b\right] d t+\left[D \varphi\left(\beta^{j}, \gamma^{j}\right)+\sigma\right] d W_{j}(t), \\
d \alpha^{j} & =\left[A \alpha^{j}+B \varphi\left(\beta^{j}, \gamma^{j}\right)+F \mathbb{E} \alpha^{j}+b\right] d t+\left[D \varphi\left(\beta^{j}, \gamma^{j}\right)+\sigma\right] d W_{j}(t), \\
d \beta^{j} & =-\left[A^{T} \beta^{j}-Q\left(\alpha^{j}-\mathbb{E} \alpha^{j}\right)\right] d t+\gamma^{j} d W_{j}(t), \\
y^{i}(0) & =y^{j}(0)=\alpha^{j}(0)=x, \quad \beta^{j}(T)=-G\left(\alpha^{j}(T)-\mathbb{E} \alpha^{j}(T)\right),
\end{aligned}\right.
$$

where $y^{(N)}=\frac{1}{N} \sum_{i=1}^{N} y^{i}$. The wellposedness of above system is easily to obtain. To prove $\left(\bar{u}_{1}, \bar{u}_{2}, \ldots, \bar{u}_{N}\right)$ is an $\epsilon$-Nash equilibrium, we need to show that for $1 \leq i \leq N$,

$$
\inf _{u_{i} \in \mathcal{U}_{a d}^{i}} \mathcal{J}_{i}\left(u_{i}, \bar{u}_{-i}\right) \geq \mathcal{J}_{i}\left(\bar{u}_{i}, \bar{u}_{-i}\right)-\epsilon .
$$

Then we only need to consider the perturbation $u_{i} \in \mathcal{U}_{a d}^{d, i}$ such that $\mathcal{J}_{i}\left(u_{i}, \bar{u}_{-i}\right) \leq \mathcal{J}_{i}\left(\bar{u}_{i}, \bar{u}_{-i}\right)$. Thus we have

$$
\mathbb{E} \int_{0}^{T}\left\langle R u_{i}(t), u_{i}(t)\right\rangle d t \leq \mathcal{J}_{i}\left(u_{i}, \bar{u}_{-i}\right) \leq \mathcal{J}_{i}\left(\bar{u}_{i}, \bar{u}_{-i}\right) \leq J_{i}\left(\bar{u}_{i}\right)+O\left(\frac{1}{\sqrt{N}}\right),
$$

which implies that

$$
\mathbb{E} \int_{0}^{T}\left|u_{i}(t)\right|^{2} d t \leq C_{0}
$$

where $C_{0}$ is a constant independent of $N$. Then similar to Lemma 3.1, we can show that there exists a constant $C_{0}$ independent of $N$ such that

$$
\sup _{1 \leq i \leq N} \mathbb{E} \sup _{0 \leq t \leq T}\left|y^{i}(t)\right|^{2} \leq C_{0}
$$

Now, for the $i^{\text {th }}$ agent, we consider the perturbation in the Problem (LCC). We introduce the following system of the decentralized limiting state with perturbation control $(j \neq i)$ :

$$
\left\{\begin{aligned}
d \bar{y}^{i} & =\left[A \bar{y}^{i}+B u_{i}+F \mathbb{E} \alpha^{i}+b\right] d t+\left[D u_{i}+\sigma\right] d W_{i}(t), \\
d \alpha^{j} & =\left[A \alpha^{j}+B \varphi\left(\beta^{j}, \gamma^{j}\right)+F \mathbb{E} \alpha^{j}+b\right] d t+\left[D \varphi\left(\beta^{j}, \gamma^{j}\right)+\sigma\right] d W_{j}(t), \\
d \beta^{j} & =-\left[A^{T} \beta^{j}-Q\left(\alpha^{j}-\mathbb{E} \alpha^{j}\right)\right] d t+\gamma^{j} d W_{j}(t), \\
\bar{y}^{i}(0) & =\alpha^{j}(0)=x, \quad \beta^{j}(T)=-G\left(\alpha^{j}(T)-\mathbb{E} \alpha^{j}(T)\right) .
\end{aligned}\right.
$$

We have the following results:

lemma 2 Lemma 3.5

$$
\mathbb{E} \sup _{0 \leq t \leq T}\left|y^{(N)}(t)-\mathbb{E} \alpha^{i}(t)\right|^{2}=O\left(\frac{1}{N}\right)
$$

lemma 2 estim 
Proof By (20), we get

$$
\left\{\begin{aligned}
d y^{(N)}= & {\left[(A+F) y^{(N)}+\frac{1}{N} B u_{i}+\frac{1}{N} \sum_{j=1, j \neq i}^{N} B \varphi\left(\beta^{j}, \gamma^{j}\right)+b\right] d t } \\
& +\frac{1}{N} \sum_{j=1}^{N} \sigma d W_{j}(t)+\frac{1}{N} D u_{i} d W_{i}(t)+\frac{1}{N} \sum_{j=1, j \neq i}^{N} D \varphi\left(\beta^{j}, \gamma^{j}\right) d W_{j}(t), \\
y^{(N)}(0)= & x .
\end{aligned}\right.
$$

Let us denote $\Pi:=y^{(N)}-\mathbb{E} \alpha^{i}$, and recall (15) which is

$$
\left\{\begin{array}{l}
d\left(\mathbb{E} \alpha^{i}\right)=\left[A \mathbb{E} \alpha^{i}+\mathbb{E} B \varphi\left(\beta^{i}, \gamma^{i}\right)+F \mathbb{E} \alpha^{i}+b\right] d t, \\
\mathbb{E} \alpha^{i}(0)=x
\end{array}\right.
$$

we have

$$
\left\{\begin{aligned}
& d \Pi=\left.(A+F) \Pi+\frac{1}{N} B u_{i}+\left(\frac{1}{N} \sum_{j=1, j \neq i}^{N} B \varphi\left(\beta^{j}, \gamma^{j}\right)-\mathbb{E} B \varphi\left(\beta^{j}, \gamma^{j}\right)\right)\right] d t \\
& \frac{1}{N} \sum_{j=1}^{N} \sigma d W_{j}(t)+\frac{1}{N} D u_{i} d W_{i}(t)+\frac{1}{N} \sum_{j=1, j \neq i}^{N} D \varphi\left(\beta^{j}, \gamma^{j}\right) d W_{j}(t), \\
& \Pi(0)=0 .
\end{aligned}\right.
$$

By the Cauchy-Schwartz inequality as well as the BDG inequality, we obtain that there exists a constant $C_{0}$ independent of $N$ which may vary line by line such that, for any $t \in[0, T]$,

$$
\begin{aligned}
\mathbb{E} \sup _{0 \leq s \leq t}|\Pi(s)|^{2} & \leq C_{0} \mathbb{E} \int_{0}^{t}\left(|\Pi(s)|^{2}+\frac{1}{N^{2}}\left|u_{i}(s)\right|^{2}\right) d s \\
& +C_{0} \mathbb{E} \int_{0}^{t}\left|\frac{1}{N} \sum_{j=1, j \neq i}^{N} \varphi\left(\beta^{j}(s), \gamma^{j}(s)\right)-\mathbb{E} \varphi\left(\beta^{j}(s), \gamma^{j}(s)\right)\right|^{2} d s \\
& +\frac{C_{0}}{N^{2}} \mathbb{E} \sum_{j=1}^{N} \int_{0}^{t}|\sigma(s)|^{2} d s \\
& +\frac{C_{0}}{N^{2}} \mathbb{E} \int_{0}^{t}\left|u_{i}(s)\right|^{2} d s+\frac{C_{0}}{N^{2}} \mathbb{E} \sum_{j=1, j \neq i}^{N} \int_{0}^{t} \mid \varphi\left(\beta^{j}(s),\left.\gamma^{j}(s)\right|^{2} d s .\right.
\end{aligned}
$$

On the one hand, by denoting $\mu(s):=\mathbb{E} \varphi\left(\beta^{j}(s), \gamma^{j}(s)\right)$ (note that since $\left(\alpha^{j}, \beta^{j}, \gamma^{j}\right), 1 \leq j \leq N$, $j \neq i$, are independent identically distributed, thus $\mu$ is independent of $j$ ), we have

$$
\begin{aligned}
& \mathbb{E}\left|\frac{1}{N} \sum_{j=1, j \neq i}^{N} \varphi\left(\beta^{j}(s), \gamma^{j}(s)\right)-\mu(s)\right|^{2} \\
\leq & 2 \mathbb{E}\left|\frac{1}{N} \sum_{j=1, j \neq i}^{N} \varphi\left(\beta^{j}(s), \gamma^{j}(s)\right)-\frac{N-1}{N} \mu(s)\right|^{2}+2 \mathbb{E}\left|\frac{1}{N} \mu(s)\right|^{2} \\
= & 2 \frac{(N-1)^{2}}{N^{2}} \mathbb{E}\left|\frac{1}{N-1} \sum_{j=1, j \neq i}^{N} \varphi\left(\beta^{j}(s), \gamma^{j}(s)\right)-\mu(s)\right|^{2}+\frac{2}{N^{2}} \mathbb{E}|\mu(s)|^{2} .
\end{aligned}
$$


Then, due to the fact that $\left(\beta^{i}, \gamma^{i}\right), 1 \leq i \leq N$ are identically distributed and $\varphi\left(\beta^{i}, \gamma^{i}\right) \in$ $L_{\mathbb{F}^{i}}^{2}(0, T ; \Gamma)$, similarly to Lemma 3.2 we can obtain that there exists a constant $C_{0}$ independent of $N$ such that

$$
\begin{aligned}
& \int_{0}^{t} \mathbb{E}\left|\frac{1}{N} \sum_{j=1, j \neq i}^{N} \varphi\left(\beta^{j}(s), \gamma^{j}(s)\right)-\mathbb{E} \varphi\left(\beta^{j}(s), \gamma^{j}(s)\right)\right|^{2} \\
\leq & \frac{C_{0}(N-1)^{2}}{N^{2}} \int_{0}^{t} \mathbb{E}\left|\frac{1}{N-1} \sum_{j=1, j \neq i}^{N} \varphi\left(\beta^{j}(s), \gamma^{j}(s)\right)-\mu(s)\right|^{2} d s+\frac{C_{0}}{N^{2}} \int_{0}^{t} \mathbb{E}|\mu(s)|^{2} d s \\
= & \frac{C_{0}(N-1)}{N^{2}} \int_{0}^{t} \mathbb{E}\left|\varphi\left(\beta^{j}(s), \gamma^{j}(s)\right)-\mu(s)\right|^{2} d s+\frac{C_{0}}{N^{2}} \int_{0}^{t} \mathbb{E}|\mu(s)|^{2} d s \\
= & O\left(\frac{1}{N}\right) .
\end{aligned}
$$

In addition, due to (21) and (22), we get

$$
\frac{C_{0}}{N^{2}} \mathbb{E} \int_{0}^{t}\left|u_{i}(s)\right|^{2} d s+\frac{C_{0}}{N^{2}} \mathbb{E} \sum_{j=1}^{N} \int_{0}^{t}|\sigma(s)|^{2} d s=O\left(\frac{1}{N}\right) .
$$

and similarly, since $\left(\beta^{j}, \gamma^{j}\right), 1 \leq j \leq N, j \neq i$, are identically distributed, we have

$$
\frac{C_{0}}{N^{2}} \mathbb{E} \sum_{j=1, j \neq i}^{N} \int_{0}^{t} \mid \varphi\left(\beta^{j}(s),\left.\gamma^{j}(s)\right|^{2} d s=O\left(\frac{1}{N}\right) .\right.
$$

Therefore, from above estimates, we get from (26) that, for any $t \in[0, T]$,

$$
\mathbb{E} \sup _{0 \leq s \leq T}|\Pi(s)|^{2} \leq C_{0} \mathbb{E} \int_{0}^{t}|\Pi(s)|^{2} d s+O\left(\frac{1}{N}\right) .
$$

Finally, by using Gronwall's inequality, we complete the proof.

lemma 3 Lemma 3.6

$$
\mathbb{E} \sup _{0 \leq t \leq T}\left|y_{t}^{i}-\bar{y}_{t}^{i}\right|^{2}=O\left(\frac{1}{N}\right)
$$

lemma 3 estim

Proof From respectively the first equation of (20) and (23), we obtain

$$
\left\{\begin{aligned}
d\left(y^{i}-\bar{y}^{i}\right) & =\left[A\left(y^{i}-\bar{y}^{i}\right)+F\left(y^{(N)}-\mathbb{E} \alpha^{i}\right)\right] d t, \\
y^{i}(0)-\bar{y}^{i}(0) & =0 .
\end{aligned}\right.
$$

With the help of classical estimates of SDE, Gronwall's inequality and (24) of Lemma 3.5, it is easily to obtain (27). The proof is completed.

lemma 4 Lemma 3.7 For all $1 \leq i \leq N$, for the perturbation control $u_{i}$, we have

$$
\left|\mathcal{J}_{i}\left(u_{i}, \bar{u}_{-i}\right)-J_{i}\left(u_{i}\right)\right|=O\left(\frac{1}{\sqrt{N}}\right)
$$


Proof Recall (2), (3) and (7), we have

$$
\begin{aligned}
& \mathcal{J}_{i}\left(u_{i}, \bar{u}_{-i}\right)-J_{i}\left(u_{i}\right) \\
= & \frac{1}{2} \mathbb{E}\left[\int_{0}^{T}\left(\left\langle Q(t)\left(y^{i}(t)-y^{(N)}(t)\right), y^{i}(t)-y^{(N)}(t)\right\rangle-\left\langle Q(t)\left(\bar{y}^{i}(t)-\mathbb{E} \alpha^{i}(t)\right), \bar{y}^{i}(t)-\mathbb{E} \alpha^{i}(t)\right\rangle\right) d t\right. \\
& \left.+\left\langle G\left(y^{i}(T)-y^{(N)}(T)\right), y^{i}(T)-y^{(N)}(T)\right\rangle-\left\langle G\left(\bar{y}^{i}(T)-\mathbb{E} \alpha^{i}(T)\right), \bar{y}^{i}(T)-\mathbb{E} \alpha^{i}(T)\right\rangle\right] .
\end{aligned}
$$

Using Lemma 3.5 and Lemma 3.6 as well as $\mathbb{E} \sup _{0 \leq t \leq T}\left(\left|\bar{y}^{i}(t)\right|^{2}+\left|\alpha^{i}(t)\right|^{2}\right) \leq C_{0}$, for some constant $C_{0}$ independent of $N$ which may vary line by line in the following, we have

$$
\begin{aligned}
& \mid \mathbb{E}\left[\int_{0}^{T}\left(\left\langle Q(t)\left(y^{i}(t)-y^{(N)}(t)\right), y^{i}(t)-y^{(N)}(t)\right\rangle-\left\langle Q(t)\left(\bar{y}^{i}(t)-\mathbb{E} \alpha^{i}(t)\right), \bar{y}^{i}(t)-\mathbb{E} \alpha^{i}(t)\right\rangle\right) d t \mid\right. \\
& \leq C_{0} \int_{0}^{T} \mathbb{E}\left|y^{i}(t)-y^{(N)}(t)-\left(\bar{y}^{i}(t)-\mathbb{E} \alpha^{i}(t)\right)\right|^{2} d t \\
& +C_{0} \int_{0}^{T} \mathbb{E}\left[\left|y^{i}(t)-y^{(N)}(t)-\left(\bar{y}^{i}(t)-\mathbb{E} \alpha^{i}(t)\right)\right| \cdot\left|\bar{y}^{i}(t)-\mathbb{E} \alpha^{i}(t)\right|\right] d t \\
& \leq C_{0} \int_{0}^{T} \mathbb{E}\left|y^{i}(t)-\bar{y}^{i}(t)\right|^{2} d t+C_{0} \int_{0}^{T} \mathbb{E}\left|y^{(N)}(t)-\mathbb{E} \alpha^{i}(t)\right|^{2} d t \\
& +C_{0} \int_{0}^{T}\left(\mathbb{E}\left|y^{i}(t)-y^{(N)}(t)-\left(\bar{y}^{i}(t)-\mathbb{E} \alpha^{i}(t)\right)\right|^{2}\right)^{\frac{1}{2}}\left(\mathbb{E}\left|\bar{y}^{i}(t)-\mathbb{E} \alpha^{i}(t)\right|^{2}\right)^{\frac{1}{2}} d t \\
& \leq C_{0} \int_{0}^{T} \mathbb{E}\left|y^{i}(t)-\bar{y}^{i}(t)\right|^{2} d t+C_{0} \int_{0}^{T} \mathbb{E}\left|y^{(N)}(t)-\mathbb{E} \alpha^{i}(t)\right|^{2} d t \\
& +C_{0} \int_{0}^{T}\left(\mathbb{E}\left|y^{i}(t)-\bar{y}^{i}(t)\right|^{2}+\mathbb{E}\left|y^{(N)}(t)-\mathbb{E} \alpha^{i}(t)\right|^{2}\right)^{\frac{1}{2}} d t \\
& =O\left(\frac{1}{\sqrt{N}}\right) \text {. }
\end{aligned}
$$

With similar argument, we can show that

$$
\begin{aligned}
& \left|\mathbb{E}\left[\left\langle G\left(y^{i}(T)-y^{(N)}(T)\right), y^{i}(T)-y^{(N)}(T)\right\rangle-\left\langle G\left(\bar{y}^{i}(T)-\mathbb{E} \alpha^{i}(T)\right), \bar{y}^{i}(T)-\mathbb{E} \alpha^{i}(T)\right\rangle\right]\right| \\
= & O\left(\frac{1}{\sqrt{N}}\right) .
\end{aligned}
$$

The proof is completed by noticing (25).

Proof of Theorem 3.1: Now, we consider the $\epsilon$-Nash equilibrium for $\mathcal{A}_{i}$ for Problem (CC). Combining Lemma 3.4 and Lemma 3.7, we have

$$
\mathcal{J}_{i}\left(\bar{u}_{i}, \bar{u}_{-i}\right)=J_{i}\left(\bar{u}_{i}\right)+O\left(\frac{1}{\sqrt{N}}\right) \leq J_{i}\left(u_{i}\right)+O\left(\frac{1}{\sqrt{N}}\right)=\mathcal{J}_{i}\left(u_{i}, \bar{u}_{-i}\right)+O\left(\frac{1}{\sqrt{N}}\right) .
$$

Consequently, Theorem 3.1 holds with $\epsilon=O\left(\frac{1}{\sqrt{N}}\right)$.

\section{Appendix}

For the readers' convenient, let us recall the following properties of projection $\mathbf{P}_{\Gamma}$ onto a closed convex set, see [2], Chapter 5. 
Theorem 4.1 For a nonempty closed convex set $\Gamma \subset \mathbb{R}^{m}$, for every $x \in \mathbb{R}^{m}$, there exists a unique $x^{*} \in \Gamma$, such that

$$
\left|x-x^{*}\right|=\min _{y \in \Gamma}|x-y|=: \operatorname{dist}(x, \Gamma) .
$$

Moreover, $x^{*}$ is characterized by the property

$$
x^{*} \in \Gamma, \quad\left\langle x^{*}-x, x^{*}-y\right\rangle \leq 0 \quad \forall y \in \Gamma .
$$

projection ch

The above element $x^{*}$ is called the projection of $x$ onto $\Gamma$ and is denoted by $\mathbf{P}_{\Gamma}[x]$.

From above theorem, it is easy to show that

Propsition 4.1 Let $\Gamma \subset \mathbb{R}^{m}$ be a nonempty closed convex set, then we have

$$
\left|\mathbf{P}_{\Gamma}[x]-\mathbf{P}_{\Gamma}[y]\right|^{2} \leq\left\langle\mathbf{P}_{\Gamma}[x]-\mathbf{P}_{\Gamma}[y], x-y\right\rangle .
$$

projection in

Proof From (28), we have

$$
\left\langle\mathbf{P}_{\Gamma}[x]-x, \mathbf{P}_{\Gamma}[x]-z\right\rangle \leq 0 \quad \forall z \in \Gamma .
$$

projection 1

and

$$
\left\langle\mathbf{P}_{\Gamma}[y]-y, \mathbf{P}_{\Gamma}[y]-z\right\rangle \leq 0 \quad \forall z \in \Gamma .
$$

projection 2

Choosing $z=\mathbf{P}_{\Gamma}[y]$ in (30) and $z=\mathbf{P}_{\Gamma}[x]$ in (31), then adding the corresponding inequalities, we obtain

$$
\left\langle\mathbf{P}_{\Gamma}[x]-x, \mathbf{P}_{\Gamma}[x]-\mathbf{P}_{\Gamma}[y]\right\rangle+\left\langle\mathbf{P}_{\Gamma}[y]-y, \mathbf{P}_{\Gamma}[y]-\mathbf{P}_{\Gamma}[x]\right\rangle \leq 0,
$$

which yields obviously

$$
\left|\mathbf{P}_{\Gamma}[x]-\mathbf{P}_{\Gamma}[y]\right|^{2} \leq\left\langle\mathbf{P}_{\Gamma}[x]-\mathbf{P}_{\Gamma}[y], x-y\right\rangle
$$

Propsition 4.2 Let $\Gamma \subset \mathbb{R}^{m}$ be a nonempty closed convex set, then the projection $\mathbf{P}_{\Gamma}$ does not increase the distance, i.e.

$$
\left|\mathbf{P}_{\Gamma}[x]-\mathbf{P}_{\Gamma}[y]\right| \leq|x-y| \text {. }
$$

projection li

Proof From (29), we have

$$
\left|\mathbf{P}_{\Gamma}[x]-\mathbf{P}_{\Gamma}[y]\right|^{2} \leq\left\langle\mathbf{P}_{\Gamma}[x]-\mathbf{P}_{\Gamma}[y], x-y\right\rangle \leq\left|\mathbf{P}_{\Gamma}[x]-\mathbf{P}_{\Gamma}[y]\right| \cdot|x-y|,
$$

which gives directly (32).

Now let us consider $\mathbb{R}^{m}$ and the projection $\mathbf{P}_{\Gamma}$ both with the norm $\|\cdot\|_{R_{0}}:=\left\langle R_{0}^{\frac{1}{2}} \cdot, R_{0}^{\frac{1}{2}} \cdot\right\rangle$, from (29), we have

Propsition 4.3 Let $\Gamma \subset \mathbb{R}^{m}$ be a nonempty closed convex set, then

$$
\left\langle\left\langle\mathbf{P}_{\Gamma}[x]-\mathbf{P}_{\Gamma}[y], x-y\right\rangle\right\rangle=\left\langle R^{\frac{1}{2}}\left(\mathbf{P}_{\Gamma}[x]-\mathbf{P}_{\Gamma}[y]\right), R^{\frac{1}{2}}(x-y)\right\rangle \geq 0 .
$$




\section{References}

[1] A. Bensoussan, J. Frehse, and S. Yam. Mean Field Games and Mean Field Type Control Theory. Springer, New York, 2013.

[2] H. Brezis. Functional Analysis, Sobolev Spaces and Partial Differential Equations. Springer, New York, 2011.

[3] P. Cardaliaguet. Notes on Mean Field Games, 2012.

[4] R. Carmona and F. Delarue. Probabilistic analysis of mean-field games. SIAM Journal on Control and Optimization, 51, 2705-2734 (2013).

[5] R. Carmona, J. P. Fouque, and L. H. Sun. Mean field games and systemic risk. Communications in Mathematical Sciences, 13, 911-933 (2015).

[6] O. Guéant, J. M. Lasry and P. L. Lions. Mean field games and applications. Paris-Princeton Lectures on Mathematical Finance 2010. Lecture Notes in Mathematics, 205-266 (2011).

[7] Y. Hu and X. Y. Zhou. Constrained stochastic LQ control with random coefficients, and application to portfolio selection. SIAM Journal on Control and Optimization, 44, 444-466 (2005).

[8] Y. Hu and S. Peng. Solutions of forward-backward stochastic differential equations. Probability Theory and Related Fields, 103, 273-283 (1995).

[9] M. Huang. Large population LQG games involving a major player: The Nash certainty equivalence principle. SIAM Journal on Control and Optimization, 48, 3318-3353 (2010).

[10] M. Huang, P. E. Caines and R. P. Malhamé. Distributed multi-agent decision-making with partial observations: asymptotic Nash equilibria. Proceedings of the 17th International Symposium on Mathematical Theory of Networks and Systems, Kyoto, Japan (2006).

[11] M. Huang, P. E. Caines, and R. P. Malhamé. Large-population cost-coupled LQG problems with non-uniform agents: Individual-mass behavior and decentralized $\varepsilon$-Nash equilibria. IEEE Trans. Automat. Control, 52, 1560-1571 (2007).

[12] M. Huang, R. P. Malhamé and P. E. Caines. Large population stochastic dynamic games: closed-loop McKean-Vlasov systems and the Nash certainty equivalence principle. Communication in Information and Systems, 6, 221-251 (2006).

[13] J. M. Lasry and P. L. Lions. Mean field games. Japan J. Math., 2, 229-260 (2007).

[14] S. L. Nguyen, M. Huang. Linear-quadratic-Gaussian mixed games with continuumparameterized minor players. SIAM Journal on Control and Optimization, 50, 2907-2937 (2012).

[15] S. Peng and Z. Wu. Fully coupled forward-backward stochastic differential equations and applications to optimal control. SIAM Journal on Control and Optimization, 37, 825-843 (1999).

[16] H. Tembine, Q. Zhu, T. Basar. Risk-sensitive mean-field games. IEEE Trans. Automat. Control, 59, 835-850 (2014).

[17] B. Wang, J. Zhang. Mean field games for large-population multiagent systems with Markov jump parameters. SIAM Journal on Control and Optimization, 50, 2308-2334 (2012). 
[18] J. Yong. Linear-quadratic optimal control problem for mean-field stochastic differential equations. SIAM Journal on Control and Optimization, 51, 2809-2838 (2013).

[19] J. Yong and X. Y. Zhou. Stochastic controls: Hamiltonian systems and HJB equations. Springer-Verlag, New York (1999). 